\title{
A case of small undifferentiated intramucosal gastric cancer with lymph node metastasis
}

\author{
Junichirou Nasu ${ }^{1}$, Shinichiro Hori ${ }^{1}$, Akinori Asagi ${ }^{1}$, Tomohiro Nishina ${ }^{1}$, Yoshio Ikeda $^{1}$, Masahito Tanimizu ${ }^{1}$, \\ Haruo Iguchi ${ }^{1}$, Kenjiro Aogi ${ }^{1}$, Akira Kurita ${ }^{2}$, and Rieko Nishimura ${ }^{3}$ \\ ${ }^{1}$ Department of Internal Medicine, National Hospital Organization Shikoku Cancer Center, 160 Minamiumemotomachi-Kou, Matsuyama \\ 791-0280, Japan \\ ${ }^{2}$ Department of Surgery, National Hospital Organization Shikoku Cancer Center, Matsuyama, Japan \\ ${ }^{3}$ Department of Clinical Laboratory, National Hospital Organization Shikoku Cancer Center, Matsuyama, Japan
}

\begin{abstract}
Early gastric cancer (EGC) has a favorable prognosis after surgical gastrectomy. For intramucosal EGC with little risk of lymph node metastasis, endoscopic mucosal resection (EMR) is an accepted treatment method. Herein we document a noteworthy case of small undifferentiated gastric cancer with nodal metastasis. A 60-year-old Japanese woman underwent gastrectomy with D2 lymph node dissection for the treatment of EGC in the lower gastric body. Histological examination revealed that signet-ring cell carcinoma was located in approximately one-third of the superficial portion of the mucosal layer, with a tumor size of $13 \mathrm{~mm}$. No lymphatic invasion, venous invasion, or fibrosis was observed in the submucosal layer. This case had nodal metastasis and was finally diagnosed as stage IB (T1N1M0) according to the Japanese Classification of Gastric Carcinoma (JCGC). The patient is alive without recurrence 6 years after treatment.
\end{abstract}

Key words Early gastric cancer - Lymph node metastasis · Undifferentiated carcinoma

\section{Introduction}

Early gastric cancer (EGC) is defined as a gastric cancer localized to the mucosa or submucosa regardless of lymph node metastasis. Radical gastrectomy with regional lymphadenectomy is the gold-standard treatment for patients with EGC [1]. For intramucosal EGC with little risk of lymph node metastasis, endoscopic mucosal resection (EMR) has been accepted as a minimally invasive treatment modality. EMR is generally indicated for intramucosal differentiated adenocarcinoma less than $20 \mathrm{~mm}$ in diameter and without ulceration [2,3]. Recently, several institutions have suggested that the indications for EMR should be expanded to

Offprint requests to: J. Nasu

Received: October 20, 2009 / Accepted: May 19, 2010 include larger, differentiated intramucosal adenocarcinoma and undifferentiated adenocarcinoma less than $20 \mathrm{~mm}$ in diameter and without ulceration.

\section{Case report}

A 60-year-old Japanese woman visited a local hospital because of anorexia and body weight loss, and gastroscopy revealed gastric cancer. She was admitted to our hospital for the treatment of gastric cancer in October 2001. She had no history of malignant disease and no family history of cancer. Upper gastrointestinal endoscopy revealed irregular, depressed mucosa, type 0 IIc in accordance with the Japanese classification of gastric carcinoma (JCGC) [4] in the large curvature of the lower gastric body (Fig. 1). The diameter was approximately $1 \mathrm{~cm}$, and there were no ulcer findings. Endoscopic ultrasonography revealed this to be an intramucosal cancer, and computed tomography examination showed no distinct lymph node metastasis. Pathologically, signet-ring cell carcinoma was detected in the biopsy specimen. Laboratory results were within normal limits, and serum carcinoembryonic antigen (CEA) levels were normal. Wedge resection of the stomach with resection of a single lymph node (\#4d) was performed in November 2001 (Fig. 2). Histological examination revealed that signet-ring cell carcinoma was located in approximately one-third of the superficial portion of the mucosal layer and that carcinoma cells had not invaded into the muscularis mucosa (Fig. $3 \mathrm{~A}, \mathrm{~B})$. The tumor was $13 \mathrm{~mm}$ in size. No lymphatic invasion, venous invasion (immunohistochemical staining with CD34 and D2-40), or ulcer scar was observed in the submucosal layer. Histologically, there was no fibrosis in the submucosal layer and no breakdown of the muscularis mucosa. The resection margin was clear of tumor cells. The single resected lymph node had metastatic cancer cells; these were also signet-ring cell car- 

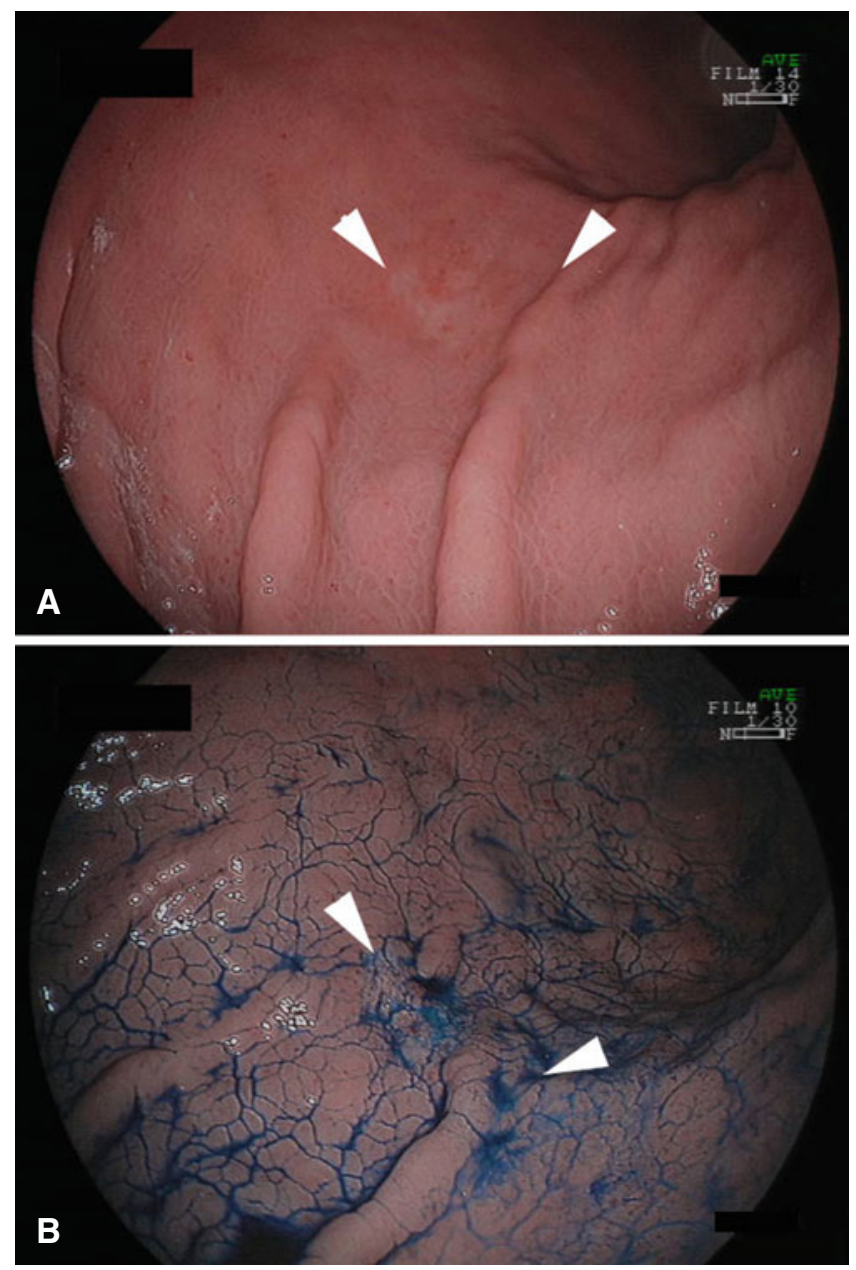

Fig. 1A, B. Gastroscopy revealed a depressed lesion in the large curvature of the lower gastric body (arrowheads). There were no ulcer findings, and endoscopic ultrasonography showed that this was an intramucosal cancer. A Without dye; B dyeing with indigo carmine

cinoma. Immediately, additional distal gastrectomy with D2 lymph node dissection (regional lymph node dissection of all group 1 and group 2 nodes) was performed, with a Billroth-I reconstruction. The gastric body with the first resection scar was histologically examined and there was no cancer lesion. Cancer cell metastasis was detected in nodes \#4sb, \#4d, and \#6 (5/65), and this case was finally diagnosed as stage IB (T1N1M0) according to the JCGC. This patient is alive without recurrence 6 years after treatment.

\section{Discussion}

For intramucosal EGC with little risk of lymph node metastasis, EMR is accepted as a minimally invasive treatment modality. Undifferentiated intramucosal EGC is known to be more commonly associated with

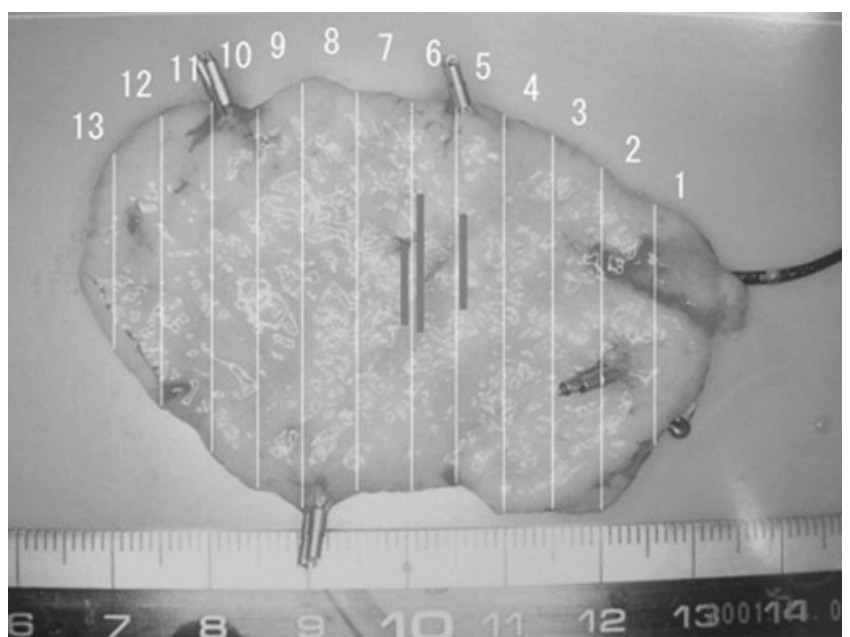

Fig. 2. Surgical specimen from partial gastrectomy. The locations of tumor cells are shown as bold lines. The tumor was $13 \mathrm{~mm}$ in diameter
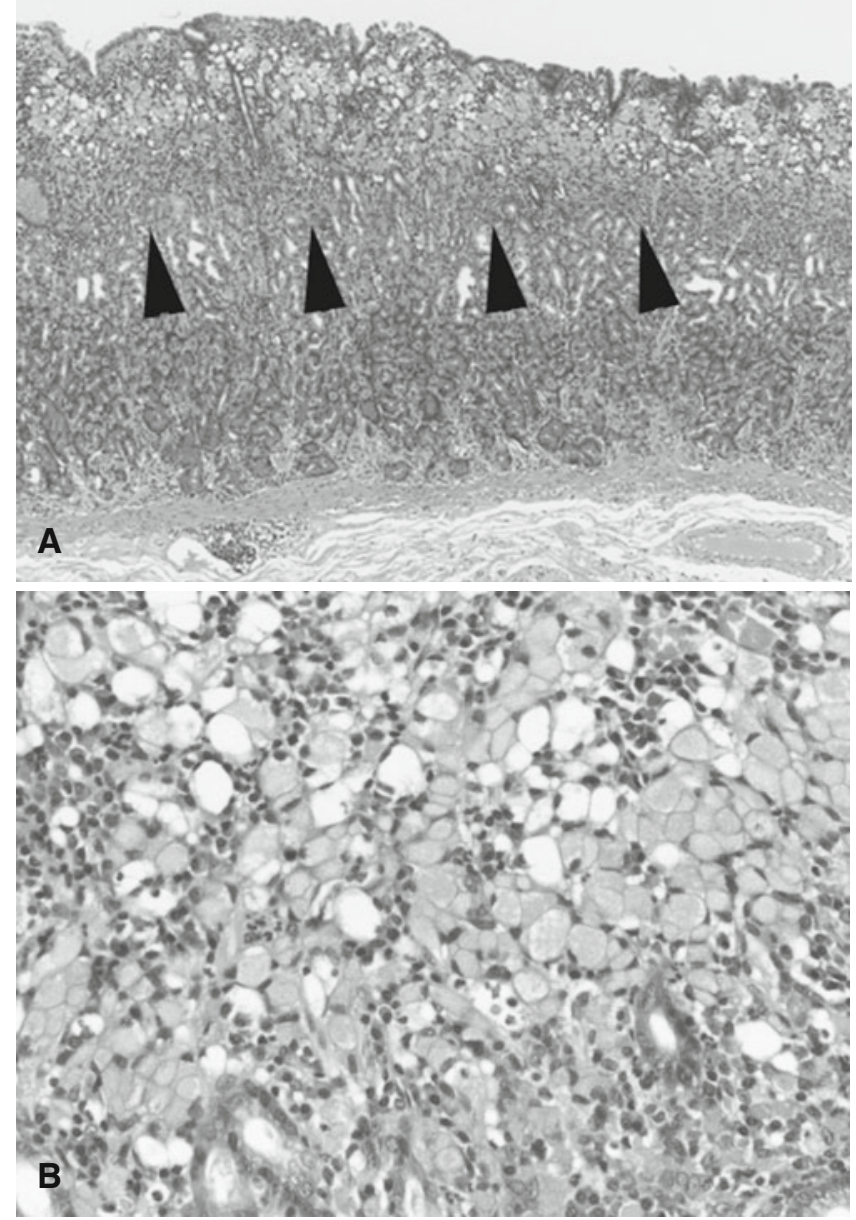

Fig. 3A, B. Histological examination. A Signet-ring cell carcinoma was located in approximately one-third of the superficial portion of the mucosal layer, and carcinoma cells had not invaded into the muscularis mucosa. B Higher magnification microscopic findings. 
lymph node metastasis compared to differentiated intramucosal EGC [5, 6], and the indications for EMR are limited to differentiated intramucosal EGC that is less than $20 \mathrm{~mm}$ and without ulceration. Gotoda et al. [3] reported that none of 141 patients with undifferentiated intramucosal EGC less than $20 \mathrm{~mm}$ in size without ulceration had lymph node metastasis. The upper limit of the $95 \%$ confidence interval was $2.6 \%$, which was considered to be sufficiently large to exclude the possibility of lymph node metastasis. Some predictive factors for lymph node metastasis in undifferentiated EGC have been reported [7-11]. Previously, we retrospectively examined factors predictive of lymph node metastasis in 332 patients with undifferentiated EGC who underwent gastrectomy, including the present patient. Multivariate analysis showed that the presence of lymphatic-vascular involvement was significantly correlated with the incidence of lymph node metastasis [8]. Abe et al. [7] reported that multivariate logistic regression analysis indicated that tumor size and lymphatic involvement were independent risk factors for lymph node metastasis. Abe et al. reported small undifferentiated intramucosal EGC cases with tumors 10, 12, and $20 \mathrm{~mm}$ in diameter without ulceration that had lymph node metastasis. These investigators concluded that EMR should not be indicated for undifferentiated EGC more than $10 \mathrm{~mm}$ in diameter.

Fewer lymphatic-vascular vessels are present in the mucosal layer than in the submucosal layer. D2-40 immunohistochemical staining has revealed that lymphatic vessels are most densely distributed in the muscularis mucosa layer of the gastric wall [12]. The cancer cells in the patient reported here were located in onethird of the mucosal layer and did not contact the muscularis mucosa. The process of cancer metastasis in this patient is difficult to conjecture. Circulating cancer cells may have played a role, as cancer cells have been identified at the DNA level in the peripheral blood of patients with EGC [13].

The expanded criteria of EMR for undifferentiated EGC are controversial. For further clarification of this issue, prognostic data for patients with undifferentiated EGC treated by EMR are required.
Acknowledgments This study was supported in part by a Grant-in-Aid for Cancer Research (21-2) from the Japanese Ministry of Health, Labour and Welfare.

\section{References}

1. Okamura T, Tsujitani S, Korenaga D, Haraguchi M, Baba H, Hiramoto Y, et al. Lymphadenectomy for cure in patients with early gastric cancer and lymph node metastasis. Am J Surg 1988;155: 476-80.

2. Yamao T, Shirao K, Ono H, Kondo H, Saito D, Yamaguchi H, et al. Risk factors for lymph node metastasis from intramucosal gastric carcinoma. Cancer 1996;77:602-6.

3. Gotoda T, Yanagisawa A, Sasako M, Ono H, Nakanishi Y, Shimoda $\mathrm{T}$, et al. Incidence of lymph node metastasis from early gastric cancer: estimation with a large number of cases at two large centers. Gastric Cancer 2000;3:2 19-25.

4. Japanese Gastric Cancer Association. Japanese classification of gastric carcinoma - 2nd English edition - Gastric Cancer 1998;1:10-24.

5. Wu CY, Chen JT, Chen GH, Yeh HZ. Lymph node metastasis in early gastric cancer: a clinicopathological analysis. Hepatogastroenterology 2002;49:1465-8.

6. Hyung WJ, Cheong JH, Kim J, Chen J, Choi SH, Noh SH. Application of minimally invasive treatment for early gastric cancer. $\mathrm{J}$ Surg Oncol 2004;85:181-5; discussion 6.

7. Abe N, Watanabe T, Sugiyama M, Yanagida O, Masaki T, Mori T, et al. Endoscopic treatment or surgery for undifferentiated early gastric cancer? Am J Surg 2004;188:181-4.

8. Nasu J, Nishina T, Hirasaki S, Moriwaki T, Hyodo I, Kurita A, et al. Predictive factors of lymph node metastasis in patients with undifferentiated early gastric cancers. J Clin Gastroenterol 2006; 40:412-5.

9. Li C, Kim S, Lai JF, Oh SJ, Hyung WJ, Choi WH, et al. Risk factors for lymph node metastasis in undifferentiated early gastric cancer. Ann Surg Oncol 2008;15:764-9.

10. Ye BD, Kim SG, Lee JY, Kim JS, Yang HK, Kim WH, et al. Predictive factors for lymph node metastasis and endoscopic treatment strategies for undifferentiated early gastric cancer. J Gastroenterol Hepatol 2008;23:46-50.

11. Park YD, Chung YJ, Chung HY, Yu W, Bae HI, Jeon SW, et al. Factors related to lymph node metastasis and the feasibility of endoscopic mucosal resection for treating poorly differentiated adenocarcinoma of the stomach. Endoscopy 2008;40:7-10.

12. Sako A, Kitayama J, Ishikawa M, Yamashita H, Nagawa H. Impact of immunohistochemically identified lymphatic invasion on nodal metastasis in early gastric cancer. Gastric Cancer 2006;9:295-302.

13. Koga T, Tokunaga E, Sumiyoshi Y, Oki E, Oda S, Takahashi I, et al. Detection of circulating gastric cancer cells in peripheral blood using real time quantitative RT-PCR. Hepatogastroenterology 2008:55:1131-5. 